\title{
Une télécommande pour les environnements immersifs
}

\author{
StÉPHANE WEIBEL ${ }^{\mathrm{a}}$ \\ IFP - Institut Français du Pétrole, 1 et 4 avenue de Bois-Préau, 92852 Rueil-Malmaison Cedex, France
}

Reçu le 30 juin 2003, accepté le 20 octobre 2003

\begin{abstract}
Résumé - Les espaces de visualisation immersive se développent dans de nombreux secteurs de la recherche et de l'industrie où ils s'intègrent de plus en plus dans les chaînes de décision, de développement ou de production. Se pose alors la question délicate de l'utilisation optimale des applications logicielles dans ces environnements. Dans cette étude, nous définirons tout d'abord une séance de travail immersive et ses contraintes en termes d'interaction. Nous passerons ensuite en revue les nombreuses solutions matérielles et logicielles déjà proposées pour repousser les limites du modèle du clavier et de la souris. Puis, à partir d'une synthèse des besoins industriels de commande des applications, nous proposerons d'imiter un modèle d'interacteur très usuel qui a fait ses preuves en utilisation domestique : la télécommande. Nous présenterons enfin le prototype que nous avons implémenté sur la base d'un assistant personnel numérique de type PocketPC connecté à un réseau radio sans fil et la bibliothèque de classes $\mathrm{C}++$ associée qui permet de l'utiliser très rapidement dans n'importe quelle application.
\end{abstract}

Mots clés : Immersif / télécommande / assistant personnel numérique / coopératif

Abstract - A remote control for immersive environments. Immersive visualization workspaces are developping in many areas of industry and research, where they are getting more and more used in workflows like decision-making, developpement or production. The issue still remains of using software applications in these environments. In this study, we will first define the notion of immersive work session, then we will list the main solutions proposed in previous work to replace the model of keyboard and mouse, trying to point out their lacks or limits. We will focus our attention on a very common and widespread interface for usual appliances, the remote control and show that it could fit a lot of industrial needs for using applications in immersive workspaces. A prototype has been developped using a PDA connected to a wireless network and a set of $\mathrm{C}++$ classes is available to implement it in any kind of application.

Key words: Immersive / remote control / personnal digital assistant / cooperative

\section{Introduction}

\subsection{La séance de travail immersive}

L'immersion visuelle se définit par l'occupation maximale du champ de vision par l'image de l'objet d'étude, rendue possible soit avec des dispositifs de types casques ou lunettes immersives soit avec des écrans de grande taille, éventuellement munis de dispositifs de vision en relief. Dans ce dernier cas, qui est celui qui nous intéresse en priorité, nous supposerons que l'utilisateur est en situation immersive s'il utilise un moyen d'affichage de l'information de même taille ou plus grand que lui, placé à faible distance. Ainsi définie, l'immersion visuelle se traduit par la possibilité de visualiser n'importe quel type de données au plus proche, et au moins à taille humaine. Qu'il s'agisse d'une galaxie de plusieurs années-lumières

\footnotetext{
a Auteur correspondant : stephane.weibel@ifp.fr
}

ou d'une molécule de quelques angströms, le phénomène est représenté par une image dont la taille est accessible par le corps tout entier. Une situation immersive pourra être créée dans un dispositif de type CAVE [1] comme le $\mathrm{SAS}^{3}$ [2], dans une salle immersive comme l'Explorium [3] ou éventuellement avec une table de travail immersive comme le Workbench [4]. Dans la suite, nous parlerons de session de travail immersive pour désigner une réunion de personnes en situation immersive. Cette étude, qui provient d'observations de séances de travail dans l'Explorium de l'IFP est plus pertinente pour les équipements de type salles immersives, même si elle est également applicable aux autres environnements immersifs.

\subsection{Contraintes pour l'interaction}

L'utilisation d'écrans à taille humaine pose un certain nombre de problèmes d'interaction avec les applications 
qui se révèlent pénalisants pour le rendement de la session de travail. Dans une salle immersive la position debout est souvent utilisée, ce qui empêche l'emploi du clavier et de la souris. Les interfaces natives des applications sont inadaptées au grand écran et les ruptures de rythme induites par la confusion des espaces de visualisation et d'interaction grèvent l'efficacité des échanges entre les participants.

\section{Position debout}

Même s'il existe une table de travail comme dans l'Explorium, la position debout est très utilisée dans une session de travail immersive. À cela, plusieurs explications peuvent être données :

- la position debout permet de s'approcher de l'écran et de s'immerger davantage encore dans les données;

- elle permet à l'utilisateur de désigner directement certains éléments visualisés sur l'écran de façon plus explicite qu'à distance, avec un pointeur laser;

- dans la position debout devant l'écran, l'utilisateur, libre de ses mouvements, peut faire des gestes amples pour englober une zone d'intérêt ou mimer le mouvement d'un objet ou de la caméra;

- la position debout dynamise la session de travail et accentue l'impact d'une assertion : « joindre le geste à la parole».

Cependant, en position debout, si l'action de désignation visuelle est facilitée, l'interaction avec l'application informatique qui tourne sur la station de visualisation devient délicate et l'utilisation du clavier et de la souris n'est plus envisageable.

\section{Utilisation des interfaces natives}

Nombre d'utilisateurs industriels demandent à pouvoir utiliser leurs applications habituelles dans un environnement immersif.

L'affichage sur un grand écran est en soi un problème lorsqu'il s'agit d'utiliser des interfaces conçues pour une utilisation sur un moniteur. L'utilisateur perd un temps précieux à « chercher » sur l'écran les menus et les boutons qu'il a l'habitude d'utiliser. De plus, toutes ces manœuvres de repérage, qui consistent à ouvrir, déplacer ou redimensionner des fenêtres obstruent la visualisation des données pour les autres participants de la session de travail.

\section{Récupération de données individuelles}

Rekimoto [5] note que lors d'une session de travail, les participants ont besoin de récupérer des données individuelles sur le disque ou le réseau. Il note que cette recherche bloque la session de travail car l'écran d'affichage est alors mobilisé par les divers éléments d'interfaces affectés à cette recherche.

Au final, utiliser une application à l'aide des interfaces habituelles projetées sur un grand écran immersif en même temps que les données s'avère pénalisant pour le rendement d'une session de travail coopératif.

\section{Solutions existantes}

De nombreux travaux ont abordé le problème de l'interaction en situation immersive. Des solutions matérielles et logicielles ont été proposées, destinées à faciliter l'utilisation, seul ou à plusieurs, des applications informatiques dans les environnements immersifs en évitant le modèle du clavier et de la souris.

Nous proposons un tour d'horizon succinct des possibilités d'interaction qui impliquent des périphériques matériels ou des interfaces hommes-machines logicielles spécifiques, en montrant les limites de ces approches.

Nous évoquerons ensuite les travaux sur l'utilisation d'assistants personnels numériques pour contrôler les applications en situation immersive.

\subsection{Utilisation de périphériques matériels}

\subsection{1 Émulation de la souris}

Une approche naturelle pour utiliser les applications habituelles dans un environnement immersif consiste à émuler les mouvements et les boutons d'une souris à l'aide de dispositifs ne nécessitant pas de table de travail.

\section{Trackballs}

On peut remplacer la souris par un trackball, qui fonctionne sans support. Certains modèles sans fil permettent ainsi de manœuvrer le pointeur habituel de la souris en s'approchant de l'écran. Ceci requiert néanmoins une certaine dextérité et un entraînement important.

\section{Tablettes graphiques}

Les tablettes graphiques utilisées par les infographistes peuvent être manipulées en position debout sans support et permettent l'émulation de la souris et des modalités supplémentaires comme la pression du stylet. Elles peuvent être utilisées à profit dans un environnement immersif. Malheureusement, il n'existe pas, à notre connaissance, de modèle sans fil.

\section{Écrans tactiles}

Un écran tactile peut être considéré comme le mélange d'un écran et d'une tablette graphique. Grâce au développement actuel des écrans LCD, de nouveau produits ont fait leur apparition, comme le produit Cintiq de Wacom [6], qui constituent des solutions potentielles pour l'interaction en situation immersive. Les limites de cette solution sont le poids du dispositif et le câble qui demeure nécessaire pour transmettre l'information vidéo vers l'écran. 


\section{Touch Pad}

Le Touch Pad, présent sur la plupart des ordinateurs portables, émule le déplacement du pointeur de la souris à partir des mouvements d'un doigt sur une zone active. Il serait intéressant de disposer d'un Touch Pad autonome sans fil muni de 3 boutons poussoirs qui permettrait une émulation de la souris en position debout.

\subsubsection{Capteurs}

\section{Effecteur - (Wand)}

Un effecteur est un périphérique composite incluant généralement un capteur à 6 degrés de liberté, des boutons-poussoirs et éventuellement un joystick ou un trackball. Il permet de «saisir» des objets 3D et de les manipuler comme si on les tenait dans la main. Le joystick ou trackball permet en plus de se déplacer dans la scène 3D. Il s'agit du périphérique-phare pour piloter les applications spécifiquement immersives, depuis les débuts de la Réalité Virtuelle. Cependant, son utilisation pour contrôler une application standard n'est pas forcément naturelle.

\subsubsection{Reconnaissance vocale}

La reconnaissance vocale est désormais une technologie mature que l'on peut exploiter dans de nombreuses applications quotidiennes. Ainsi, certains téléphones mobiles sont munis d'un module de reconnaissance vocale qui permet d'appeler directement un correspondant, présent dans l'annuaire, par simple énoncé de son nom.

L'utilisation de la reconnaissance vocale en Réalité Virtuelle a été souvent étudiée et utilisée et cette technique s'est avérée très intéressante comme outil de contrôle d'une application immersive. Cependant, elle est généralement limitée à une utilisation individuelle nécessitant un apprentissage de la voix et donc peu adaptée à une séance de travail à plusieurs.

\subsubsection{Autres techniques}

On peut citer une technique originale de repérage spatial par action des pieds sur une carte projetée au sol [7], qui a l'avantage de laisser les mains totalement libres.

\subsection{Interfaces homme-machine logicielles}

\subsubsection{Interfaces 3D}

\section{Principe}

Les interfaces virtuelles 3D sont des objets particuliers inclus dans la scène visualisée et sur lesquels l'utilisateur peut agir pour paramétrer l'application. Ces objets sont des équivalents 3D des habituels menus, boutons, interrupteurs (toggles), ou variateurs (sliders). Cette approche permet d'unifier les interactions avec les données et avec les éléments d'interfaces, par exemple pour la sélection. Les interfaces virtuelles 3D sont généralement utilisées avec un pointeur $3 \mathrm{D}$ virtuel commandé par un effecteur équipé d'un capteur de position et d'orientation.

\section{Limites}

Les interfaces virtuelles 3D partagent l'espace d'affichage avec les données et on peut considérer que cet espace perdu est préjudiciable à l'analyse visuelle de la scène par l'utilisateur. Elles sont intégrées dans la scène au même titre que les données et partagent donc la même fréquence de rafraîchissement graphique. Si celle-ci n'est pas suffisamment soutenue $(<10 \mathrm{~Hz})$, elles deviennent rapidement inutilisables. De plus, cette approche ne permet pas facilement de changer les paramètres puis de valider.

\subsubsection{Interfaces 2D}

\section{Principe}

Dans un espace de visualisation immersive, on peut afficher sur l'écran des interfaces 2D inspirées des visions tête-haute utilisées dans les casques des pilotes. Les éléments d'interface sont réduits à leur plus simple expression pour ne pas accaparer l'espace de visualisation et ils peuvent être semi-transparents, pour laisser les données toujours visibles.

Notons que ce type d'interface est depuis longtemps utilisé avec succès dans le domaine du jeu vidéo. Les nouveaux titres de jeux vidéos en 3D temps-réel sont généralement interfacés à l'aide d'éléments 2D éventuellement semi-transparents facilement amovibles. Les interfaces 2D utilisées dans les jeux vidéos, en particulier sur console, ne reposent pas sur un modèle d'interaction avec un pointeur qui serait difficilement utilisable à l'aide d'une manette ou d'un joystick. Les options du jeu sont fixées en suivant une arborescence dont chaque niveau est composé de quelques éléments simples permettant le paramétrage et la navigation. Pour sélectionner un élément, l'utilisateur actionne généralement un pad directionnel et un bouton de sélection. Ce type d'interaction semble donner depuis longtemps satisfaction aux joueurs et permet de minimiser l'espace occupé sur l'écran par les éléments d'interface, au prix d'une utilisation qui peut sembler un peu plus laborieuse qu'avec les interfaces informatiques à pointeur.

Dans d'autres domaines, des menus 2D, superposés aux images sont utilisés dans un grand nombre d'appareils électroniques connectés à des écrans : téléviseurs, magnétoscopes, lecteurs DVD, boîtiers de réception câble ou satellite, caméscopes. Ils sont généralement manipulés à l'aide d'une télécommande qui permet une navigation simple parmi les différentes options.

C'est ce modèle d'interaction qui nous a particulièrement intéressés pour la conception de notre dispositif de contrôle des applications en environnement immersif. Nous verrons en particulier, dans la partie 4, 
en quoi le dispositif très répandu de la télécommande combine la simplicité d'utilisation avec un grand nombre de possibilités d'interactions.

\subsection{Les assistants personnels numériques}

\subsubsection{Présentation}

Un assistant personnel numérique est un petit ordinateur sans clavier muni d'un écran tactile utilisable à l'aide d'un stylet. Deux standards de faits existent : Palm Pilot et Pocket PC [8]. Un assistant personnel numérique peut être équipé d'une carte PCMCIA de communication réseau radio sans fil qui le transforme alors en objet mobile et communicant tout à fait intéressant, en particulier pour le contrôle des applications en situation immersive.

\subsubsection{Assistants personnels et Réalité Virtuelle}

L'utilisation d'assistants personnels numériques pour la Réalité Virtuelle a été évoquée dès 1993 par Fitzmaurice [9] qui simula la restitution d'un monde 3D sur un assistant personnel grâce à un ingénieux prototype développé à partir d'un téléviseur LCD miniature connecté à une station graphique. Aujourd'hui il est possible d'afficher sur un assistant personnel une image bitmap en couleur représentant une pré-visualisation des données, générée sur la station de visualisation qui tient lieu de serveur de visualisation. Cette technique est employée dans des produits comme VNC [10] ou VizServer [11]. Fitzmaurice [12] a également proposé de rendre l'assistant personnel sensible à sa position relative dans un environnement immersif en le munissant d'un capteur à 6 degrés de liberté.

Watsen [13] a montré l'intérêt d'utiliser un assistant personnel comme instrument de navigation et de paramétrage dans un environnement immersif. Il a ouvert la voie à ce type d'interaction en insistant sur la nécessité d'adapter de façon contextuelle les interfaces présentées et de pouvoir proposer plusieurs interfaces différentes pour la même action.

Hill [14] a exploité les nouveautés technologiques des assistants personnels, en particulier leur ouverture au langage Java, pour développer un cadre informatique flexible pour l'interaction avec les espaces immersifs : JAIVE.

Park [15] avec le projet Virtual Harlem a bien montré l'intérêt des assistants personnels pour se repérer rapidement dans une reconstitution virtuelle à grande échelle.

Myers [16] a démontré, dans le cadre du projet Pebbles $[17,18]$, comment l'utilisation d'un assistant personnel dans une séance de travail coopératif permettait d'éviter les ruptures de rythme induits par la recherche d'informations stockées. Un assistant personnel est pour lui un moyen idéal pour saisir du texte, par exemple pour des annotations. Il a de plus développé, sur la base d'un assistant personnel, un dispositif d'émulation des mouvements et des boutons de la souris d'un PC, le Remote Commander.

\section{Applications industrielles}

Dans cette partie, nous déterminerons les points qui nous paraissent importants pour l'utilisation « industrielle » d'une application de visualisation dans un espace de travail immersif. Ces éléments ont été collectés pendant plus d'un an par l'observation de nombreuses séances de travail menées dans l'Explorium de l'IFP par des équipes multidisciplinaires de différents secteurs industriels.

L'impression générale est la suivante : même si les performances croissantes des matériels graphiques permettent actuellement de combiner haute résolution, qualité d'image et forte interactivité, les applications industrielles habituelles ne sont pas adaptées aux environnements immersifs, ce qui pénalise lourdement le déroulement et le résultat des séances de travail.

Rappelons que la motivation des utilisateurs d'une salle de visualisation avancée comme l'Explorium provient avant tout des opportunités suivantes :

- partager les informations visuelles de façon confortable entre plusieurs participants ;

- utiliser ses applications habituelles sur une machine particulièrement « gonflée » en termes de mémoire ou de performances graphiques.

Ainsi, pendant la séance de travail, les utilisateurs vont s'employer à manipuler sur le grand écran de la salle immersive les applications qu'ils ont l'habitude d'utiliser sur leurs stations de travail. Les éléments importants qui ressortent de cette utilisation sont les suivants.

\section{Optimisation de l'espace d'affichage}

Par manque d'habitude, les utilisateurs consacrent beaucoup de temps à gérer l'occupation du grand écran par les diverses fenêtres de l'application.

\section{Traçabilité de la séance de travail}

Durant la séance de travail, un grand nombre de commentaires et de choix stratégiques peuvent être exprimés par les participants. L'archivage de ces informations est un besoin important qui n'est pas facilement mis en œuvre dans un environnement immersif. Idéalement, la collecte de ces informations devrait être couplée aux outils bureautiques habituels, afin de faciliter la rédaction de compte-rendus de séance complets et renseignés.

Les interactions concernées sont la capture d'images, les annotations textuelles, schématiques ou vocales.

\section{Permettre le travail coopératif}

L'utilisation à plusieurs d'un espace de visualisation immersive rentre dans le cadre d'étude du CSCW (Computer-Supported Cooperative Work). L'emploi concurrent des applications par plusieurs utilisateurs constitue en particulier un aspect très important dans l'efficacité d'une séance de travail immersive.

Finalement, dans les applications industrielles de la visualisation immersive, on se rend compte que des 
problèmes d'ergonomie, parfois très simples, peuvent constituer des freins importants à la productivité d'une séance de travail.

Dans la suite, nous étudierons un modèle très simple d'interaction, très utilisé et qui offre un excellent compromis entre les fonctionnalités et la facilité d'utilisation : la télécommande.

\section{La télécommande}

La télécommande est devenue un objet hautement répandu dans de nombreuses applications de la vie quotidienne. Le mot télécommande désigne une action à distance (préfixe télé, d'origine grecque qui signifie loin). Nous proposons ici une étude des particularités du modèle d'interaction sous-jacent. Nous verrons que ce modèle peut être particulièrement intéressant dans le cadre du contrôle des applications en situation immersive.

\subsection{Particularités du modèle}

Alors qu'auparavant, une télécommande ne permettait de contrôler qu'un seul modèle de téléviseur, il est aujourd'hui possible de n'en utiliser qu'une seule, dite universelle, pour tous les modèles et toutes les marques. Audelà, l'inflation du nombre d'appareils électroniques et du nombre de télécommandes associées pousse les industriels à développer une télécommande véritablement universelle qui permettra de contrôler à l'aide du même dispositif l'ensemble des appareils électroniques de la maison.

La télécommande est un dispositif « naturel» pour le grand public dès lors qu'il s'agit de commander un ensemble de fonctionnalités standard. Le modèle d'interaction repose sur la décomposition des commandes en actions unitaires simples correspondant généralement à l'appui sur un seul bouton. On peut identifier deux types d'actions.

\section{Actions directes}

Ces actions adressent directement une fonctionnalité de l'appareil sans utiliser d'affichage ou de guide visuel, par exemple pour changer de chaîne sur un téléviseur. Les boutons à employer sont généralement implicitement connus de tous : le numéro de la chaîne sur le pavé numérique ou les touches avant/arrière pour les programmes.

\section{Actions via un menu}

Ces actions sont couplées à l'affichage d'un menu qui permet de suivre les choix effectués et de visualiser les options proposées par exemple pour la programmation d'un magnétoscope, la navigation dans les menus d'un titre DVD ou l'interaction avec un boîtier numérique de réception câble ou satellite (télévision interactive). La télécommande permet d'avancer étape par étape dans les différentes options proposées. Contrairement à des interfaces avec pointage, qui fonctionnent par désignation directe des éléments, une télécommande, comme une manette de jeu vidéo, propose à l'utilisateur des actions simples de validation et d'annulation pour parcourir l'arborescence des possibilités. L'affichage de ces menus «superposés » est généralement à la fois claire et discrète, et monopolise une surface assez limitée. La simplicité de ce modèle d'interaction en a fait un standard très utilisé jusque sur les petits écrans des téléphones mobiles.

Ce mode de navigation est aussi à la base de la navigation hypertexte des débuts de l'Internet et reste très utilisé pour se déplacer à l'intérieur des sites du Web.

\subsection{Assistants personnels et télécommande}

Dans un certain nombre d'applications les assistants personnels peuvent remplacer les habituelles télécommandes à infrarouge, grâce à leur port infrarouge IrDA. Certaines applications pour assistants personnels permettent de remplacer plusieurs télécommandes d'appareils électroniques disparates par une seule interface unifiée. Ces applications sont même capables d'acquérir les codes de contrôle directement à partir des télécommandes d'origine. Au-delà, les assistants personnels constituent une évolution naturelle du modèle d'interacteur de la télécommande. Ils permettront des fonctionnalités au moins identiques couplées à une souplesse très importante, par exemple pour la mise à jour contextuelle des interfaces. Nichols $[19,20]$ a proposé de remplacer une ou plusieurs télécommandes d'appareils électroniques par un assistant personnel donnant accès à des interfaces sur mesure éventuellement fabriquées à la volée selon l'appareil en question.

Dans le domaine de la domotique, en utilisant un assistant personnel numérique, il sera possible de compiler dans un unique dispositif mobile l'ensemble des états et des actions possibles dans la maison.

\section{Dispositif}

On a vu qu'il était intéressant d'utiliser un assistant personnel connecté sans fil pour contrôler une application immersive grâce à des interfaces sur mesure et éventuellement contextuelles. C'est un dispositif particulièrement adapté à la saisie de textes et à la recherche des informations lors d'une séance de travail coopératif sur grand écran. On a également mis en évidence que le modèle d'interaction très répandu de la télécommande permet d'optimiser l'espace d'affichage, en donnant accès soit à des actions implicite directes, soit à une navigation simple dans des menus superposés qui n'occupent que très peu d'espace visuel.

Nous proposons donc d'utiliser un assistant personnel connecté au réseau par une liaison radio sans fil pour interagir avec les applications utilisées lors d'une séance de travail immersive sur grand écran à l'aide d'interfaces, simples et génériques, imitant les interactions d'une télécommande, quelles soient directes ou via un menu affiché en superposition sur l'écran. 


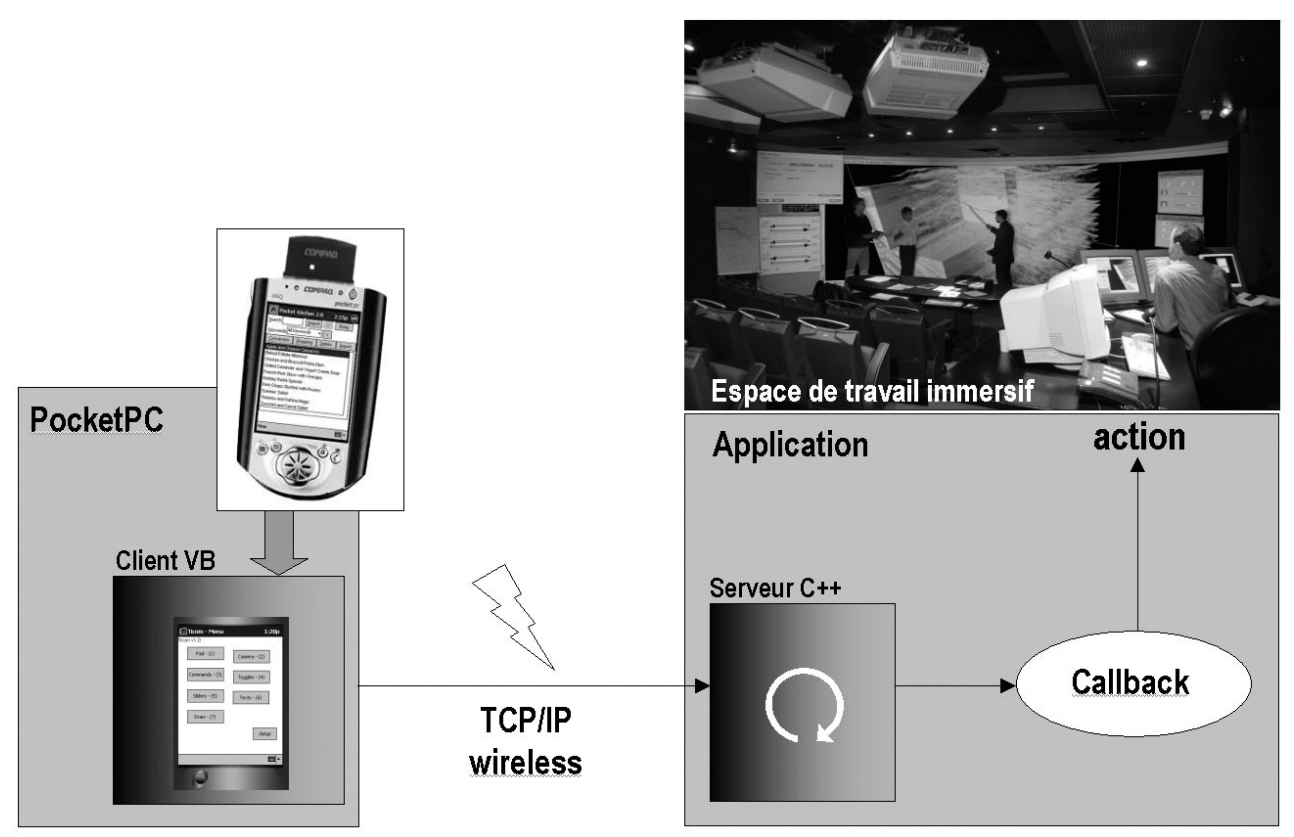

Fig. 1. Schéma de principe.

\subsection{L'interface cliente}

L'interface cliente de notre dispositif, destinée à être utilisée sur l'assistant personnel, a été développée avec Microsoft Embedded Visual Basic pour PocketPC.

Elle est composée de plusieurs pages de commandes accessibles depuis une page d'accueil. Sur chaque page, un bouton «menu» permet de revenir sur la page d'accueil. Une page particulière d'options permet de configurer le dispositif. L'interface fonctionne en mode client, elle se connecte à un serveur spécifique, lancé sur la station de visualisation par l'application à commander à l'aide de la bibliothèque de classes $\mathrm{C}++$ fournie. La page d'accueil est composée de boutons qui permettent d'accéder aux différentes pages de commandes. Quelques pages de commandes de l'interface cliente sont montrées dans la partie 6 , consacrée aux applications du dispositif.

\subsection{Le module serveur}

Le serveur est un sous-module de l'application destinée à être contrôlée par le dispositif. Il est lancé sur la station de visualisation comme un nouveau thread de l'application à commander en utilisant la bibliothèque de classes $\mathrm{C}++$ fournie. Les actions de l'utilisateur sur l'interface du PocketPC sont recueillies par l'application, qui interroge régulièrement le serveur, dans une fonction de type callback. Parmi les informations importantes récupérées par l'application figurent en particulier la page et le numéro de l'élément d'interface actionné.

\subsection{Déploiement}

Le schéma de principe du dispositif est présenté en figure 1.

\subsection{Utilisation}

L'application à commander utilise la bibliothèque de classes $\mathrm{C}++$ fournie pour instancier le serveur et lui fournir l'adresse d'une fonction callback qui va recueillir en direct toutes les informations sur les interactions menées sur le PocketPC. Par la suite, l'application est en charge d'appeler à intervalles réguliers une méthode de mise à jour du serveur qui provoque l'appel de la callback si un nouvel événement s'est produit sur le PocketPC.

\section{Applications}

Les manipulations que nous présentons ici ont été mises en places dans l'Explorium de l'IFP et testées par l'équipe d'exploitation, habituée à utiliser le clavier et la souris pour le contrôle des applications de visualisation.

\subsection{Viewer Open Inventor}

Dans cette application, on utilise la page Camera sur le PocketPC pour modifier en temps réel les paramètres de la caméra d'un viewer Open Inventor [21]. Cette page, reproduite en figure 2, fournit des éléments d'interface génériques pour manipuler un objet ou une caméra 3D. On y trouve un pad de rotation, un pad de translation et un slider de zoom.

La manipulation dans l'Explorium et l'aspect de l'interface cliente sont montrés en figure 4. Cette application exploite les capacités tactiles et graphiques du PocketPC, puisque la précision des actions dépend de la résolution des éléments d'interface. 

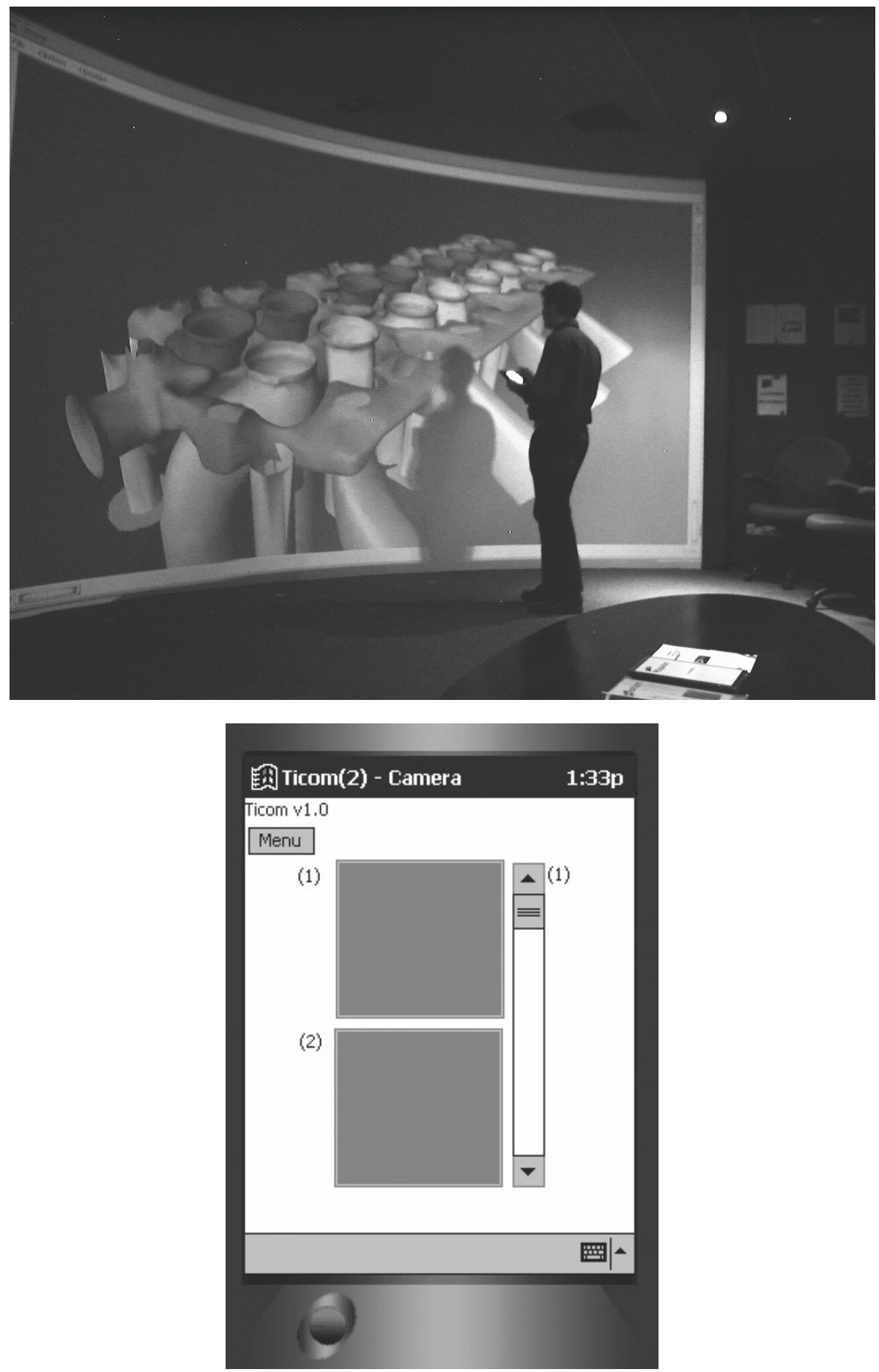

Fig. 2. Contrôle d'un viewer Open Inventor.

\subsection{Capture d'images}

Pour cette application, illustrée en figure 3 on utilise un bouton pour déclencher directement une capture d'image de l'écran complet de l'Explorium. Les images capturées sont archivées automatiquement dans un espace réservé de l'Intranet. Dans ce cas, il s'agit d'une utilisation directe de la «télécommande », équivalente à un changement de chaîne.

\subsection{Annotations}

Une zone de texte est utilisée pour saisir un message qui sera intégré directement dans l'application de visualisation. Pour saisir ce message, l'utilisateur peut, au choix, employer le clavier virtuel ou la reconnaissance d'écriture intégrée au PocketPC. On voit en figure 4 que cela permet de saisir des messages textuels en position debout. 


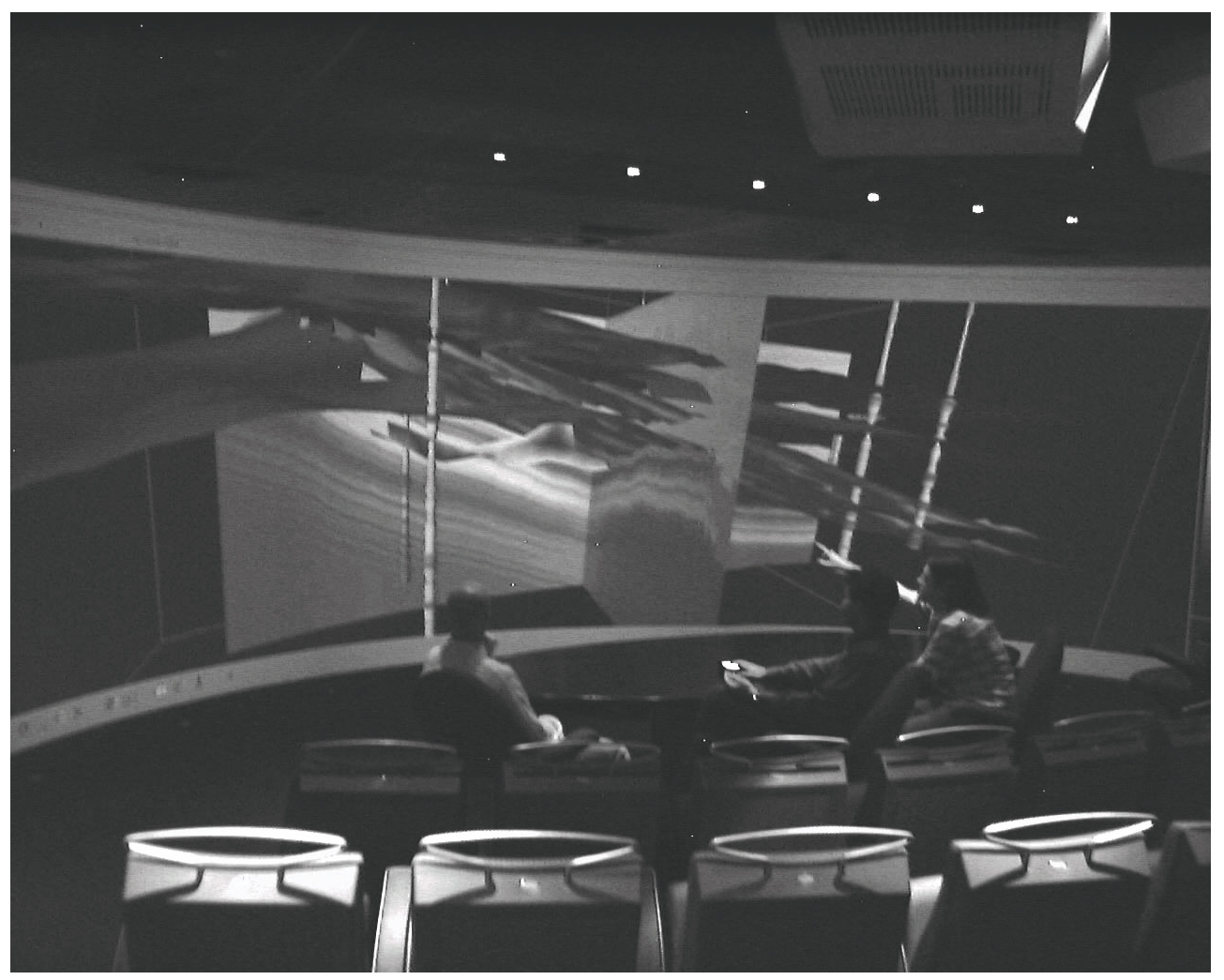

Fig. 3. Capture d'images.

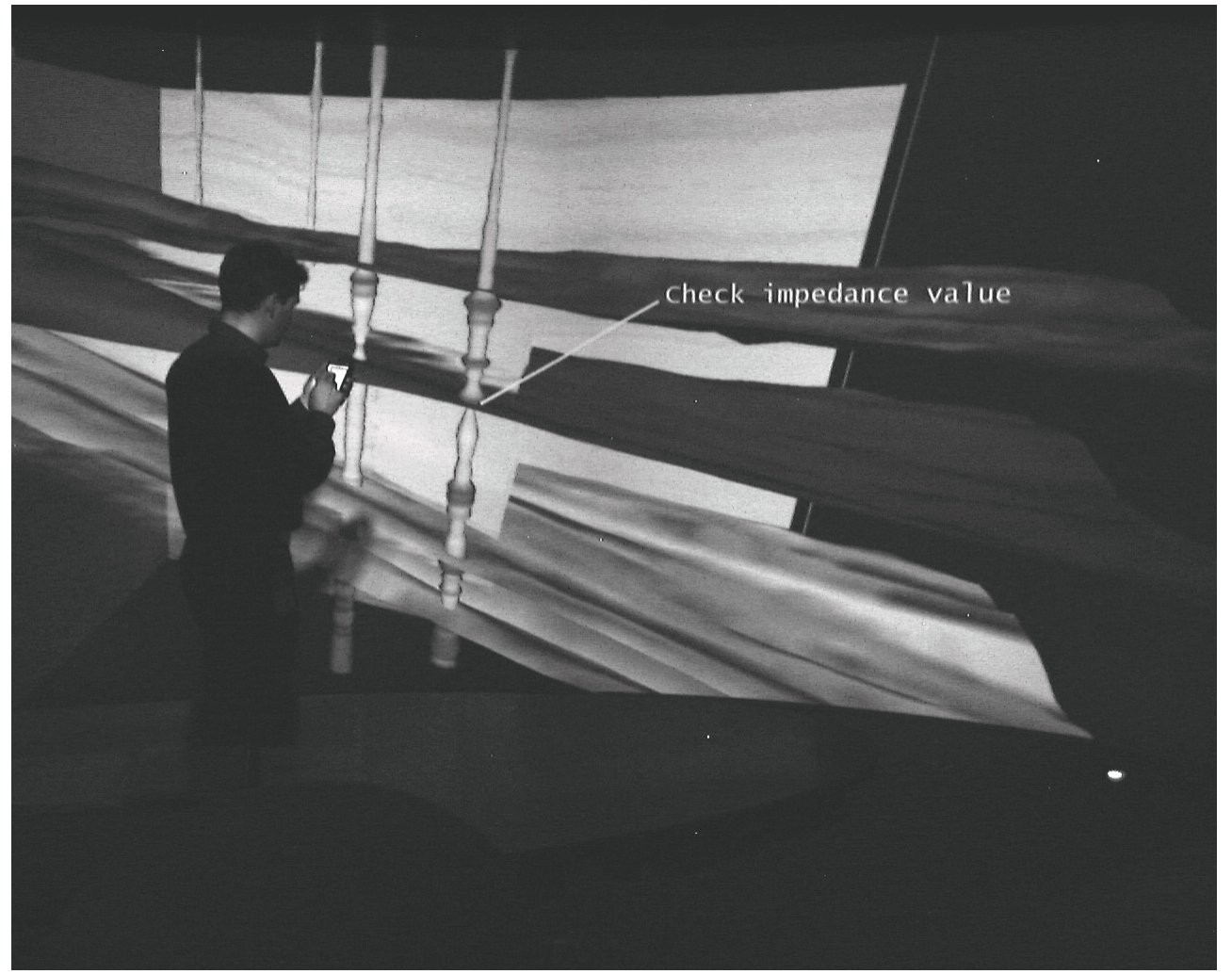

Fig. 4. Saisie d'annotations. 

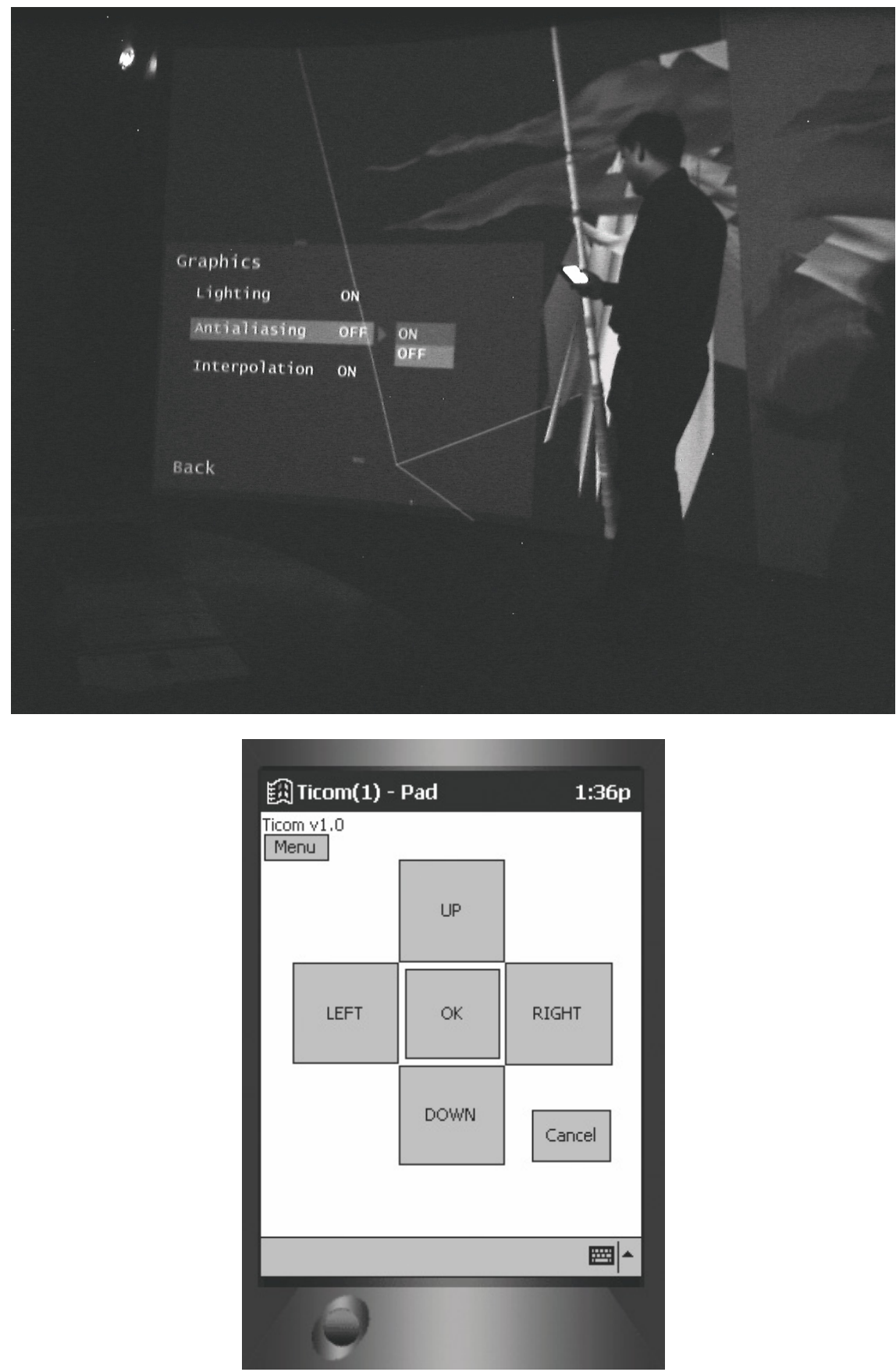

Fig. 5. Navigation dans un menu superposé.

\subsection{Options d'affichage avec menu}

La page Pad de l'interface cliente est utilisée pour naviguer dans un menu, affiché en superposition sur l'application de visualisation, qui propose différentes options de rendu graphique. La manipulation dans l'Explorium et l'aspect de l'interface cliente sont représentés en figure 5. Dans ce cas, notre dispositif est utilisé comme une télécommande de boîtier numérique câble ou satellite ou une manette de jeu vidéo.

\section{Conclusion et travaux futurs}

Nous avons développé un dispositif de contrôle sans fil des applications immersives, basé sur un assistant personnel connecté au réseau. Cette «télécommande» universelle offre, sur le même objet, une large gamme de modèles d'interaction, proposés habituellement sur différents dispositifs hétérogènes :

- actions directes par appui sur un bouton, comme une télécommande de téléviseur;

- navigation dans des menus superposés à l'écran comme pour la télévision interactive; 
- contrôle précis d'un déplacement ou d'une rotation, comme avec une souris ou une trackball;

- saisie de textes avec reconnaissance d'écriture.

Grâce à la bibliothèque de classes $\mathrm{C}++$ fournie, la mise en œuvre du dispositif est assez simple dans la plupart des applications.

Les améliorations du dispositif seront axées sur les problématiques suivantes.

\section{Contrôle complet du site}

Outre la station de visualisation, une salle immersive comme l'Explorium est équipée d'un ensemble de matériels divers pour gérer par exemple la lumière ou le son. Ces matériels sont souvent contrôlés à l'aide d'écrans tactiles fixes disposés sur la console de commande. On peut imaginer intégrer ces contrôles sur le même assistant personnel que nous utilisons pour contrôler les applications, le transformant ainsi en dispositif de contrôle complet du site.

\section{Interfaces contextuelles}

Contrairement à une télécommande de matériel domestique, qui est principalement un effecteur, un assistant personnel peut recevoir des informations en retour de la part de l'application pilotée. Ceci rend possible la configuration contextuelle de l'interface sur l'assistant en fonction de l'état de l'application pilotée. Ainsi, un élément d'interface de type «variateur » peut être affecté d'un intitulé correspondant au paramètre qu'il permet de modifier. L'application actuelle ne permet pas cette mise à jour, mais la prochaine version permettra de le faire.

\section{Utilisation avec d'autres périphériques}

L'assistant personnel peut être positionné au niveau du poignet à l'aide d'une sangle comme une montre et laisser ainsi les mains libres, pour utiliser un capteur de position et d'orientation. L'utilisateur peut ainsi sélectionner directement un objet dans la scène 3D à l'aide du capteur et l'interface d'édition personnalisée de cet objet apparaît alors sur l'assistant personnel.

Remerciements. Je remercie avant tout l'équipe de l'Explorium de l'IFP, Laurent Grizon, Timour Jgenti, Steve Travers, Mathieu Sors pour leur aide précieuse. Merci aussi à Jean-Louis Pajon, responsable du groupe Visualisation et Réalité Virtuelle de l'IFP pour ses conseils et sa relecture avisée. Je remercie également Benoit Lelandais pour avoir partagé depuis le début mon engouement pour tous ces petits objets mobiles et communiquants.

\section{Références}

[1] C. Cruz-Neira, D.J. Sandin, T.A. Defanti, Surroundscreen Projection-based Virtual Reality: The Design and Implementation of the CAVE, Proceedings of SIGGRAPH 93, Annual Conference Series, Aug. 1993, pp. $135-142$
[2] $\mathrm{SAS}^{3}:$ http://www.z-a.net/sascube

[3] Explorium : www-europe.sgi.com/services/ professionnal/success/ifp.html

[4] W. Krüger, C.A. Bohn, B. Fröhlich, H. Schüth, W. Strauss, G. Wesche, The responsive workbench: A virtual work environment. IEEE Computer, July 1995, pp. 42-48

[5] J. Rekimoto, A Multiple Device Approach for Supporting Whiteboard-based Interactions, in Proceedings SIGCHI'98 : Human Factors in Computing Systems 18-23 April 1998, Los Angeles, CA, pp. 344-351

[6] Wacom Cintiq pen display : www.wacom.com

[7] J.J. LaViola, D.A. Feliz, D.F. Keefe, R.C. Zeleznik, Hands-Free Multi-Scale Navigation in Virtual Environments, Proceedings of the 2001 Symposium on Interactive 3D Graphics, SI3D 2001, March 26-29, 2001, Chapel Hill, NC, USA

[8] HP/Compaq IPAQ : www . compaq. com/products/handhelds/pocketpc

[9] G.W. Fitzmaurice, S. Zhai, M.H. Chignell, Virtual Reality for Palmtop Computers, ACM Transactions on Information Systems, Vol. 11(3) July 1993, pp. 197-218

[10] VNC : Virtual Network Computing www.uk.research.att.com/vnc

[11] SGI VizServer : www.sgi.com/software/vizserver

[12] G.W. Fitzmaurice, W. Buxton, The Chameleon: Spatially Aware Palmtop Computer, ACM CHI'94, pp. 451-452

[13] K. Watsen, R.P. Darken, M.V. Capps, A Handheld Computer as an Interaction Device to a Virtual Environment. International Immersive Projection Technology Workshop (IPT 1999), Stuttgart - Germany, May 10-11, 1999

[14] L.C. Hill, C. Cruz-Neira, Palmtop Interaction Methods for Immersive Projection Technology Systems, Immersive Projection Technology Workshop 2000, Ames, Iowa

[15] K.S. Park, J. Leigh, A.E. Johnson, B. Carter, J. Brody, J. Sosnoski, Distance Learning Classroom Using Virtual Harlem, Proceedings of VSMM 2001, Berkeley, CA, USA, 25-27 Nov. 2001

[16] B.A. Myers, H. Stiel, R. Gargiulo, Collaboration Using Multiple PDAs Connected to a PC. Proceedings CSCW'98: ACM Conference on Computer-Suppported Cooperative Work, November 14-18, 1998, Seattle, WA, USA, pp. 285-294. http://www.cs.cmu.edu/ pebbles/

[17] B.A. Myers, K.P.L. Lie, B.-C. Yang, J. Two-Handed Input Using a PDA And a Mouse. Proceedings CHI'2000: Human Factors in Computing Systems. The Hague, The Netherlands, April 1-6, 2000 http://www.cs.cmu.edu/ pebbles/

[18] B.A. Myers, R.C. Miller, B. Bostwick, C. Evankovich, Extending the Windows Desktop Interface With Connected Handheld Computers, 4th USENIX Windows Systems Symposium, August 3-4, 2000, Seattle, WA, USA, pp. 79-88. http://www.cs.cmu.edu/ pebbles/

[19] J.W. Nichols, Using Handhelds as Controls for Everyday Appliances: A Paper Prototype Study. ACM CHI'2001 Student Posters. Seattle, WA, USA, March 31-April 5, 2001, pp. 443-444. http://www.cs.cmu.edu/ pebbles/

[20] J.W. Nichols, Generating Remote Control Interfaces for Complex Appliances, to appear in Proceedings of UIST'2002, Paris, France, Oct. 27-30

[21] Open Inventor : oss.sgi.com/projects/inventor www.tgs.com 